\title{
Design Method for Channel Diffusers of Centrifugal Compressors
}

\author{
Mykola Kalinkevych and Andriy Skoryk \\ Technical Thermophysics Department, Sumy State University, 2 Rimsky-Korsakov Street, Sumy 40007, Ukraine \\ Correspondence should be addressed to Andriy Skoryk; avsrs@mail.ru
}

Received 29 April 2013; Revised 14 July 2013; Accepted 20 July 2013

Academic Editor: Enrico Sciubba

Copyright ( 2013 M. Kalinkevych and A. Skoryk. This is an open access article distributed under the Creative Commons Attribution License, which permits unrestricted use, distribution, and reproduction in any medium, provided the original work is properly cited.

\begin{abstract}
The design method for channel diffusers of centrifugal compressors, which is based on the solving of the inverse problem of gas dynamics, is presented in the paper. The concept of the design is to provide high pressure recovery of the diffuser by assuming the preseparation condition of the boundary layer along one of the channel surfaces. The channel diffuser was designed with the use of developed method to replace the vaned diffuser of the centrifugal compressor model stage. The numerical simulation of the diffusers was implemented by means of CFD software. Obtained gas dynamic characteristics of the designed diffuser were compared to the base vaned diffuser of the compressor stage.
\end{abstract}

\section{Introduction}

The operating conditions and purpose of centrifugal compressor should be taken into account when choosing the type of its diffuser. Channel diffusers (CD) could be more preferable as compared to other types in the following cases: (1) at small flow angles at the diffuser inlet; (2) when the gas passes from the diffuser channels to the separated cameras; (3) if the diffuser channels turn to the channels of the return element of compressor.

Traditional geometry of channel diffusers includes the initial section shaped as a logarithmic spiral along one of the vane surfaces and the main section with straight walls (Figure 1(a)). The channel diffusers with wedge vanes are also widely used (Figure 1(b)).

Some authors recommend using the Reneau et al. [1] and Runstadler Jr. and Dean Jr. [2] database for the flat plane diffusers to design wedge-shaped channel diffusers. Kano et al. [3] and Clements and Artt [4] indicated that such data cannot be used to design the high-performance diffuser of centrifugal compressor because of the distorted three-dimensional swirled flow at the inlet. In addition, such a database does not cover the wide range of geometries and inlet flow conditions.
Generally, the most effective vane geometry may be obtained by solving the inverse problem of gas dynamics. The maximum pressure rise in diffuser may be achieved when the flow is close to separation. Stratford [5] obtained experimentally the flow with stable preseparation condition of the boundary layer along one of the surfaces of the twodimensional diffuser. It was shown that, by specifying the preseparation pressure distribution, the required pressure rise may be attained in the shortest possible distance and with the least possible dissipation of energy for a given diffuser dimensions and initial boundary layer. Such approach was later used by Liebeck [6] for estimation of the shape of high lift airfoil and by Hobbs and Weingold [7] for designing the high performance axial compressor airfoils. However, there is no information in applying the preseparation velocity (or pressure) distributions for the design of centrifugal compressor cascades with radial swirled flow.

Kalinkevych et al. [8] showed that efficiency of the vaned diffuser (VD) of centrifugal compressor may be improved by using such a concept. In this work the center line shape of the vanes with constant thickness was obtained by defining the preseparation velocity distribution along the pressure surface of the vane. 


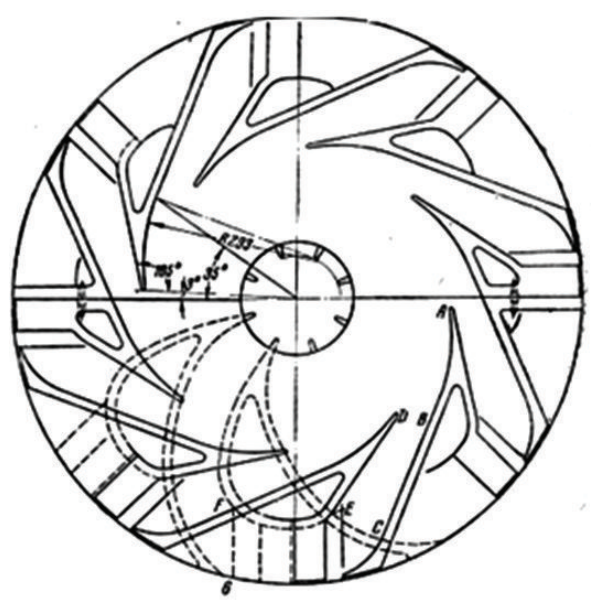

(a)

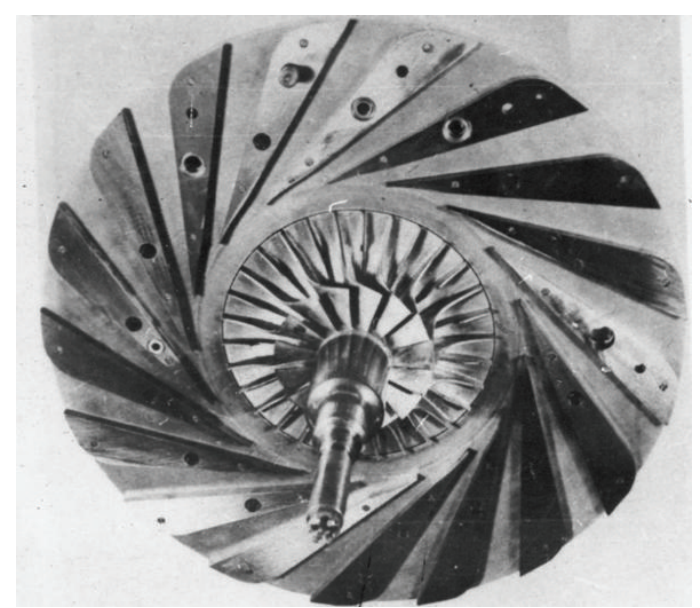

(b)

FIGURE 1: Channel diffusers of traditional geometry: (a) channel diffuser with initial section shaped as a logarithmic spiral, (b) channel diffuser with wedge vanes.

The principles of design method for high-performance channel diffusers (CD) of centrifugal compressors, in which vane thickness increases along the radius, are presented in the paper.

\section{Design Method}

According to the presented method, the diffuser design is based on assuming the preseparation condition of the boundary layer along one of the vane surfaces. Mathematical model for solving the inverse problem of gas dynamics is developed for the steady adiabatic gas flow without separations.

The angular momentum change about axis $z$ for the annular element of gas with width $\Delta b$ and mass flow rate $\Delta \bar{m}$ from the diffuser inlet $r_{\text {in }}$ to the current section $r$ (Figure 2) is as follows:

$$
\Delta M=\Delta \bar{m} \cdot\left(r_{\text {in }} \cdot c_{\text {in }} \cdot \cos \alpha_{\text {in }}-r \cdot c \cdot \cos \alpha\right) .
$$

Moment of forces acting on the $z_{v}$ vanes of diffuser from $r_{\text {in }}$ to $r$ is as follows:

$$
\Delta M=\Delta b \cdot z_{v} \cdot \int_{r_{\text {in }}}^{r} \Delta p \cdot r \cdot d r .
$$

Continuity equation is of the form

$$
\Delta \bar{m}=c_{r} \cdot \rho \cdot 2 \pi \cdot r \cdot \Delta b \cdot \tau .
$$

Equations (1), (2), and (3) may be represented using gas dynamics relations for isentropic flow:

$$
\begin{aligned}
r_{\text {in }} & \cdot \lambda_{\text {in }} \cdot \cos \alpha_{\text {in }}-r \cdot \lambda \cdot \cos \alpha \\
= & \frac{b \cdot z_{v} \cdot p_{\mathrm{in}}^{*}}{\bar{m} \cdot a_{\mathrm{cr}}} \cdot \int_{r_{\mathrm{in}}}^{r}\left[\pi\left(\lambda_{\mathrm{ps}}\right)-\pi\left(\lambda_{\mathrm{ss}}\right)\right] \cdot r \cdot d r, \\
& \bar{m}=\lambda \cdot \varepsilon(\lambda) \cdot a_{\mathrm{cr}} \cdot \rho^{*} \cdot 2 \pi \cdot r \cdot b \cdot \tau \cdot \sin \alpha,
\end{aligned}
$$

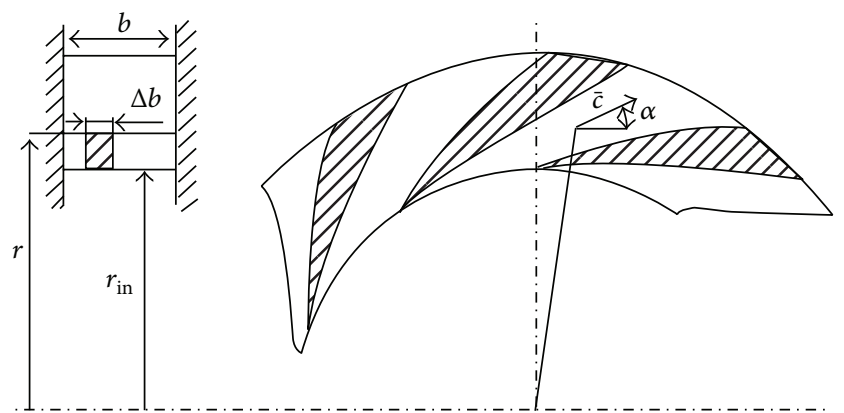

Figure 2: Channel diffuser scheme.

where $\lambda, \lambda_{\mathrm{ps}}$, and $\lambda_{\mathrm{ss}}\left(a_{\mathrm{cr}}=\sqrt{(2 \gamma /(\gamma+1)) R T^{*}}\right.$ is the critical velocity) are the mean flow velocity within the diffuser channel, velocity along the pressure surface, and velocity along the suction surface of the vane, respectively and $\alpha$ is the mean flow angle within the diffuser channel.

Pressure and density relations are determined as a function of velocity:

$$
\begin{aligned}
& \pi(\lambda)=\frac{p}{p^{*}}=\left(1-\frac{\gamma-1}{\gamma+1} \cdot \lambda^{2}\right)^{\gamma /(\gamma-1)}, \\
& \varepsilon(\lambda)=\frac{\rho}{\rho^{*}}=\left(1-\frac{\gamma-1}{\gamma+1} \cdot \lambda^{2}\right)^{1 /(\gamma-1)} .
\end{aligned}
$$

The blockage factor is given by the following equation:

$$
\tau=1-\frac{\delta^{\prime} \cdot z_{v}}{2 \pi \cdot r \cdot \sin \alpha},
$$

where $\delta^{\prime}=\delta_{v}+\sum \delta^{*}$ is the modified vane thickness; $\delta_{v}$ is vane thickness, and $\sum \delta^{*}$ is total displacement thickness of boundary layers in the vane channel estimated using the Loitcyanskii method [9]. 
Velocity distribution, which provides the preseparation condition of the boundary layer along the pressure surface of the vane, is defined by the formula [8]:

$$
\lambda_{\mathrm{ps}}=\lambda_{1} \cdot\left[1+\frac{\left(\bar{l}-\bar{l}_{1}\right) \cdot\left(2+H_{s}\right) \cdot\left(-f_{s}\right)}{\bar{\delta}_{1}^{* *}}\right]^{-1 /\left(2+H_{s}\right)} .
$$

Parameters marked with subscript "1" are the coefficients, which affect the given velocity distribution quantitatively.

The set of (4) includes unknowns $\lambda, \delta_{v}$, and $\lambda_{\mathrm{ss}}$. The dependence $\alpha=f(r)$ may be given as linear.

For linear pressure distribution along the vane pitch the relation between the velocities may be defined as

$$
\pi\left(\lambda_{\mathrm{ps}}\right)+\pi\left(\lambda_{\mathrm{ss}}\right)=2 \cdot \pi(\lambda)
$$

By substituting equation (8) into equation (4), equation (4) can be solved using numerical methods for numerical integration and root finding.

The initial data for the design are

(i) gas properties $(R, \gamma, \nu)$;

(ii) static pressure $p_{\text {in }}$ and static temperature $T_{\text {in }}$ at diffuser inlet;

(iii) inlet and outlet flow angles $\left(\alpha_{\text {in }}, \alpha_{\text {out }}\right)$;

(iv) mass flow rate;

(v) geometrics of the meridional contour;

(vi) quantity of vanes $z_{v}$;

(vii) mean flow angle distribution along the diffuser channel $(\alpha=f(r))$

As a result of calculation, the vane thickness distribution along the radius $\delta_{v}=f(r)$ is estimated, so the geometry of the vane is totally defined.

The compressor design point flow parameters at the impeller exit are used as mentioned in above initial data for the diffuser design. Presented design method is valid for the subsonic flow along the entire diffuser.

\section{Application of the Design Method for the Centrifugal Compressor Model Stage}

Using presented method, the CD for the model centrifugal compressor stage of JSC "Sumy Frunze NPO" was designed. The design was implemented for the parameters at the diffuser inlet at the design point of the stage. The parameters of the flow were obtained by numerical simulation of the base model compressor stage with VD.

The values of design parameters are shown in Table 1 . The meridional contour geometry, inlet and outlet angles of the vanes are the same as for the base VD.

The given velocity distribution for the CD design is shown in Figure 3. The geometry parameters of the vane are shown in Figure 4. Vane thickness distribution was obtained as a result of design calculation.

The relative radius in Figures 3 and 4 is defined by formula

$$
\bar{r}_{i}=\frac{r_{i}-r_{\text {in }}}{r_{\text {out }}-r_{\text {in }}} \text {. }
$$

TABLE 1: Initial data for channel diffuser design.

\begin{tabular}{lc}
\hline Design parameter & Value \\
\hline Pressure at the diffuser inlet, $\mathrm{Pa}$ & 118000 \\
Temperature at the diffuser inlet, $\mathrm{K}$ & 319 \\
Mass flow rate, $\mathrm{kg} / \mathrm{s}$ & 1.775 \\
Quantity of vanes & 17 \\
Inlet radius, $r_{3}, \mathrm{~m}$ & 0.2622 \\
Outlet radius, $r_{4}, \mathrm{~m}$ & 0.3092 \\
Width of diffuser, $b_{3}, \mathrm{~m}$ & 0.0155 \\
Vane leading edge thickness, $\mathrm{m}$ & 0.003 \\
Vane leading edge centerline angle, $\alpha_{3 v},{ }^{\circ}$ & 22 \\
Vane trailing edge centerline angle, $\alpha_{4 v},{ }^{\circ}$ & 37 \\
\hline
\end{tabular}

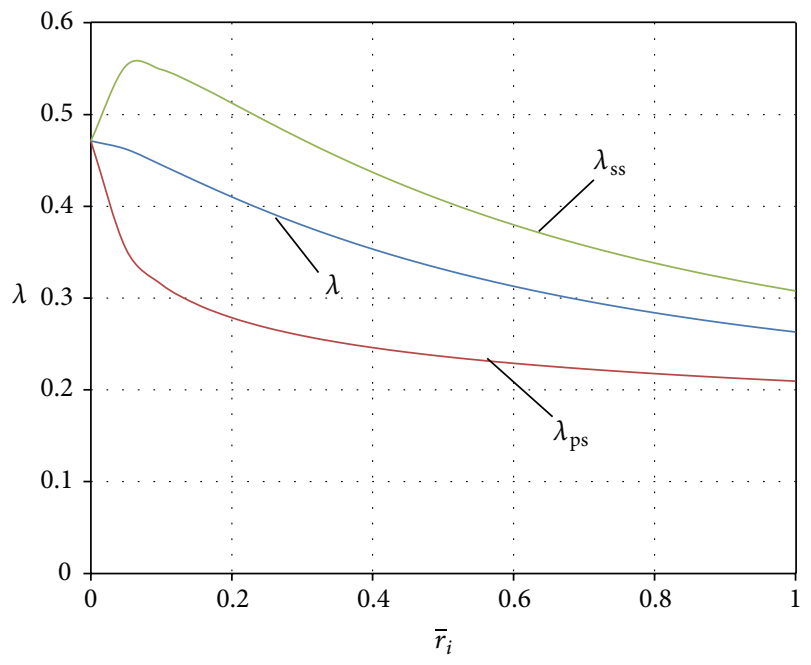

FIGURE 3: Given velocity distribution for the channel diffuser design.

The comparison between the vane channels of the base VD and designed CD is shown in Figure 5.

\section{Numerical Simulation}

Numerical simulation was performed by use of commercial CFD software ANSYS CFX v.14 for two different compressor stages. The first one is the model compressor stage of JSC "Sumy Frunze NPO" with base VD. The second stage has the same impeller as the first. The only difference is the diffuser, which was designed using presented method (see Section 3).

4.1. Grid Quality and Preprocessor Setup. The steady-state model and high resolution discretization scheme were used for simulations. SST-turbulence model is the most acceptable model for the centrifugal compressor flow simulations [10], which in the case of sufficient grid refinement shows appropriate results for the near-wall boundary layers and flow core.

The structured hexahedral grids for impeller and diffusers were created in ANSYS TurboGrid. While creating the nearwall prismatic layers, it was checked that value of $y^{+}$is less than 2 . The quantity of grid points within the boundary layers was no less than 20 . The coarser grid was created for the flow core, which is acceptable for the SST-turbulence model. The 

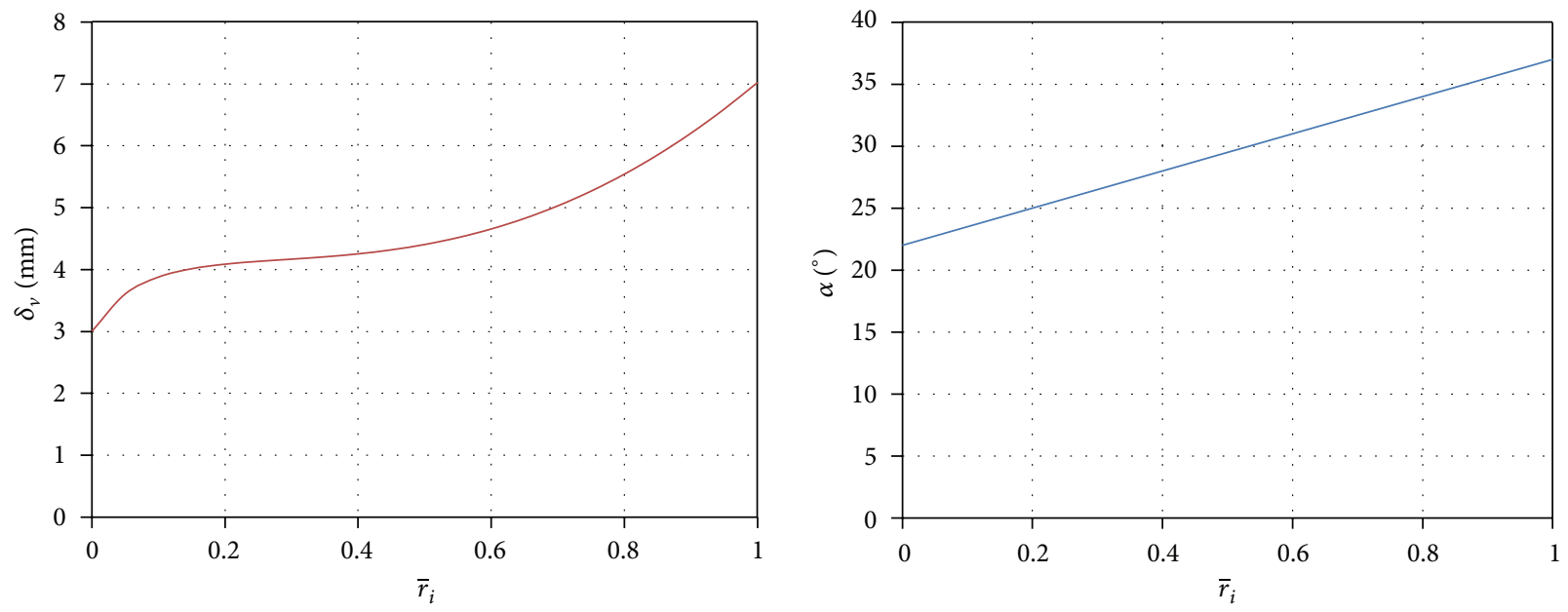

FIGURE 4: Vane thickness and angle distribution for the designed channel diffuser.

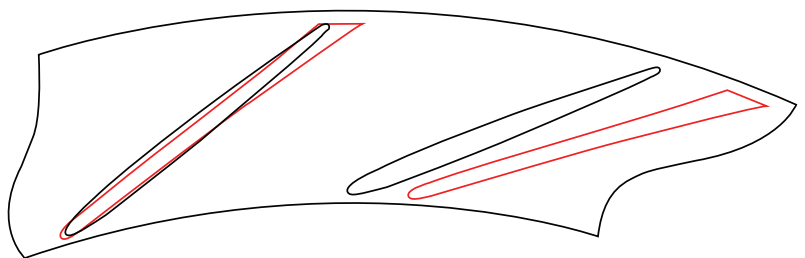

FIGURE 5: The comparison between the base VD vane channel (black) and designed CD vane channel (red).

impeller grid consists of 690690 elements (Figure 6(a)). The CD grid consists of 637296 elements (Figure 6(b)), and VD grid (Figure 6(c)) consists of 618618 elements. Due to the differences in geometry of these diffusers, it is not possible to create totally topologically identical grids. The parameters which define the topology of the near-wall prismatic layers and the quantity of grid elements in meridional plane were identical. Therefore this topological difference is acceptable for the comparison. The difference is only in the flow core elements quantity.

The main parameters specified in ANSYS CFX preprocessor are shown in Table 2. To connect the respective surfaces of the impeller and diffuser, the interface "stage" was used. This type of interface is usually used for the steady state calculations; the parameters at the interface surfaces are averaged circumferentially. Therefore interface "stage" is oriented for estimating of the integral characteristics of the compressor stage.

As the convergence criteria, the discrepancy in static pressure recovery coefficient equal to 0.01 and in total pressure loss coefficient equal to 0.005 has been used.

The grid independence study showed that for the design point the diffusers' nondimensional characteristics change is distinct for the diffusers node quantity less than 400000 . For off-design conditions the results of simulation are more gridsensitive.

The main purpose of simulations was to establish the more effective diffuser by comparing their nondimensional
TABLE 2: Boundary conditions and models, specified in ANSYS CFX preprocessor.

\begin{tabular}{ll}
\hline Boundary or model & Option \\
\hline Impeller inlet & Total pressure, total temperature \\
Diffuser outlet & Mass flow rate \\
Interface type between & Stage \\
impeller and diffuser & SST \\
Turbulence model & Total energy \\
Heat transfer model & Air ideal gas \\
Fluid model & Adiabatic \\
Wall heat transfer model & \\
\hline
\end{tabular}

characteristics. Generated grids were indicated as sufficient for such calculations.

To evaluate the diffuser aerodynamic performance, the pressure recovery coefficient

$$
C_{p}=\frac{p_{\text {out }}-p_{\text {in }}}{p_{\text {in }}^{*}-p_{\text {in }}}
$$

and total pressure loss coefficient

$$
\zeta=\frac{p_{\text {in }}^{*}-p_{\mathrm{out}}^{*}}{p_{\mathrm{in}}^{*}-p_{\text {in }}}
$$

were used.

4.2. Simulation Results. Obtained nondimensional diffusers' characteristics are shown in Figure 7 as a function of incidence angle

$$
i_{3}=\alpha_{3 v}-\alpha_{3},
$$

where $\alpha_{3 v}$ is vane centerline angle at the diffuser inlet.

It can be seen that characteristics of $\mathrm{CD}$ are better than of $\mathrm{VD}$ at the range of positive incidence angles. Moreover, maximal value of pressure recovery of $\mathrm{CD}$ is slightly higher than of VD. The shape of characteristics of CD is more flat, 

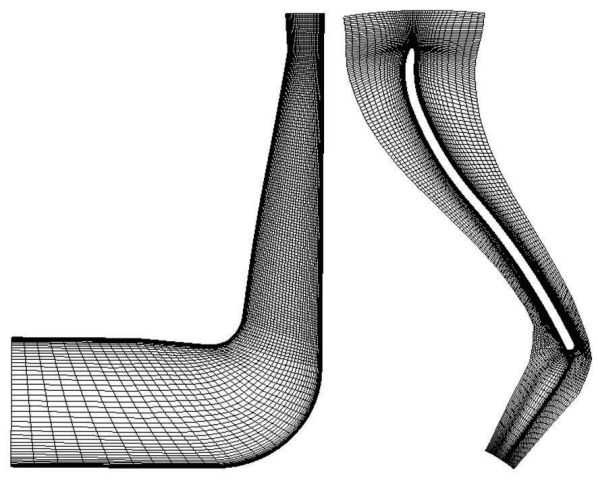

(a) Impeller

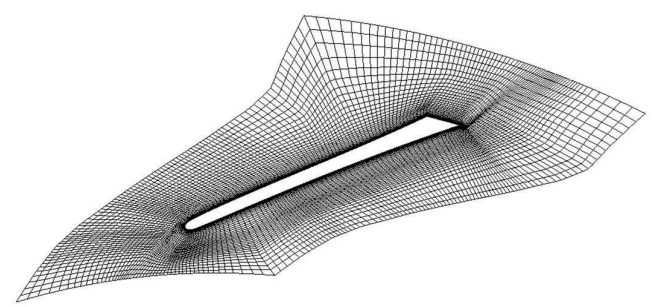

(b) Channel diffuser

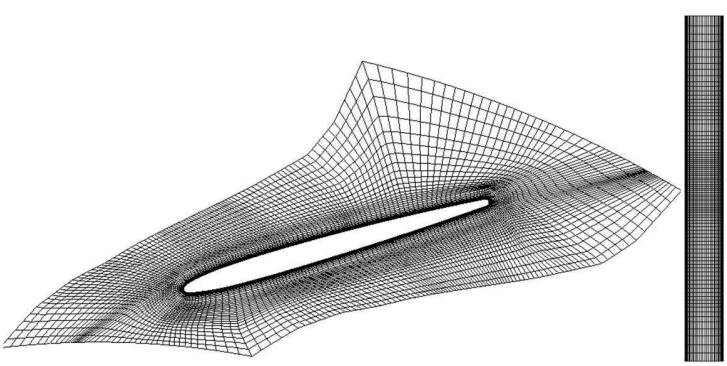

(c) Vaned diffuser

FIgURE 6: Grids of the stage elements.

especially for the total pressure loss coefficient. In order to understand these results the flow pattern within the diffusers was examined.

Figure 8 presents the velocity vectors within both diffusers for $i_{3} \approx 4^{\circ}$. Predicted high loss level and low pressure recovery of $\mathrm{VD}$ occur due to the wide flow separation region. Due to the high pressure gradients, the flow separation occurs on the suction surface of the vane and covers the area from the hub side to midspan. Reducing the effective area resulted in low pressure recovery.

The flow pattern within the $\mathrm{CD}$ is more favorable due to the controlled flow deceleration provided by the designed vane geometry. For the operating conditions range of $i_{3} \approx$ $0^{\circ}-4^{\circ}$, the total losses include only the friction loss and wake mixing loss at diffuser outlet (Figures 8 and 9). Therefore the shape of the loss characteristic of $\mathrm{CD}$ is almost straight.

As it can be seen from Figure 7 for the top pressure recovery operating condition of $\mathrm{VD}$ at $i_{3} \approx 1^{\circ}$, the pressure recovery of $\mathrm{CD}$ is slightly lower, whereas the losses are practically the same. The flow separation within the CD was detected at $i_{3} \approx 5^{\circ}$.

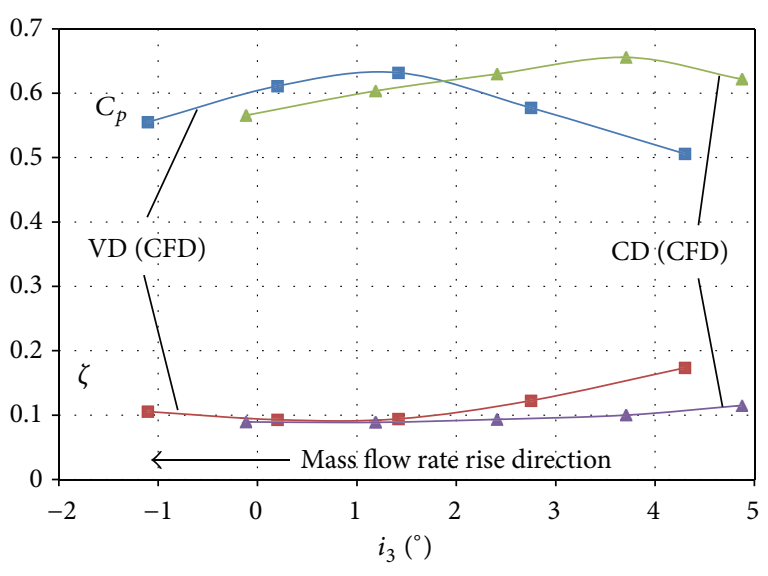

FIGURE 7: Nondimensional characteristics of designed CD and base VD.
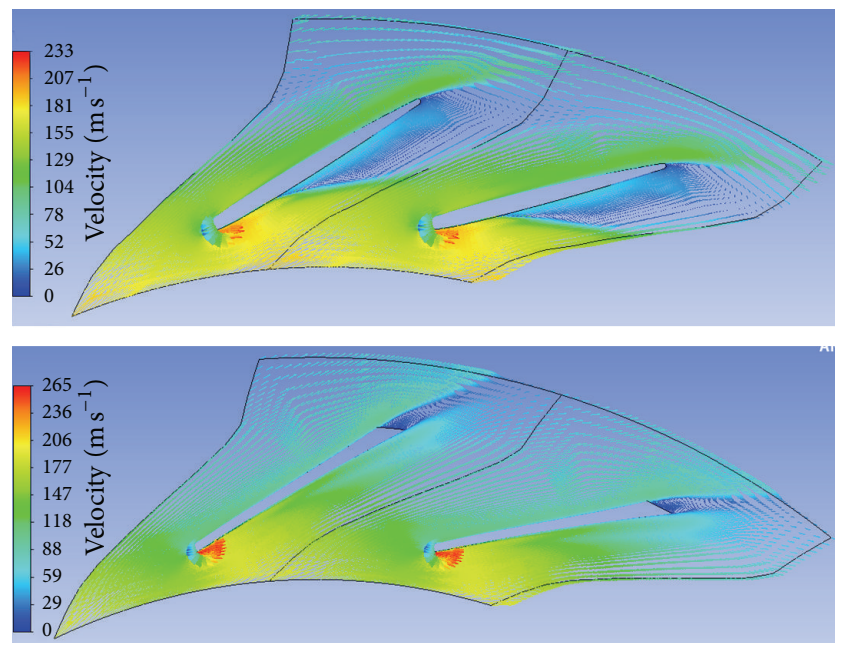

(a) Hub side
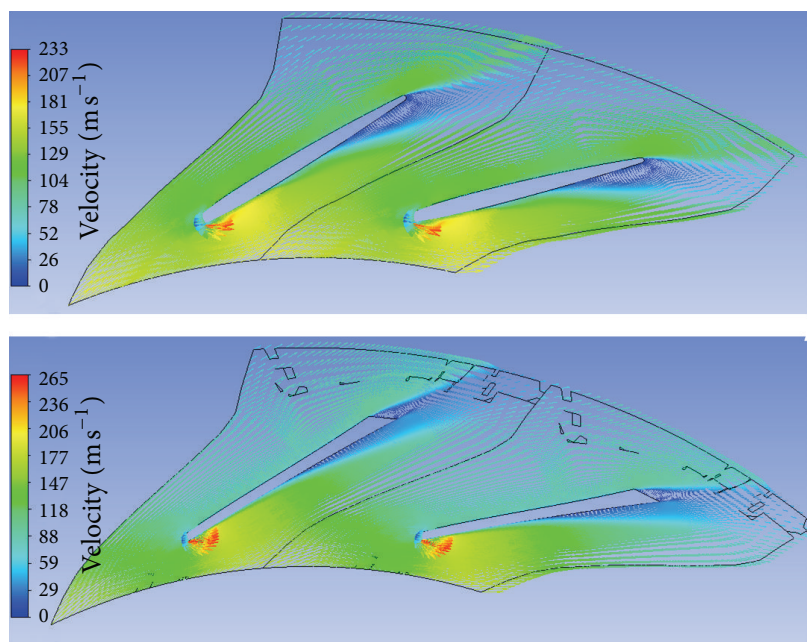

(b) Midspan

FIGURE 8: Velocity vectors for different flow surfaces of VD (top) and $\mathrm{CD}$ (bottom) at $i_{3} \approx 4^{\circ}$. 

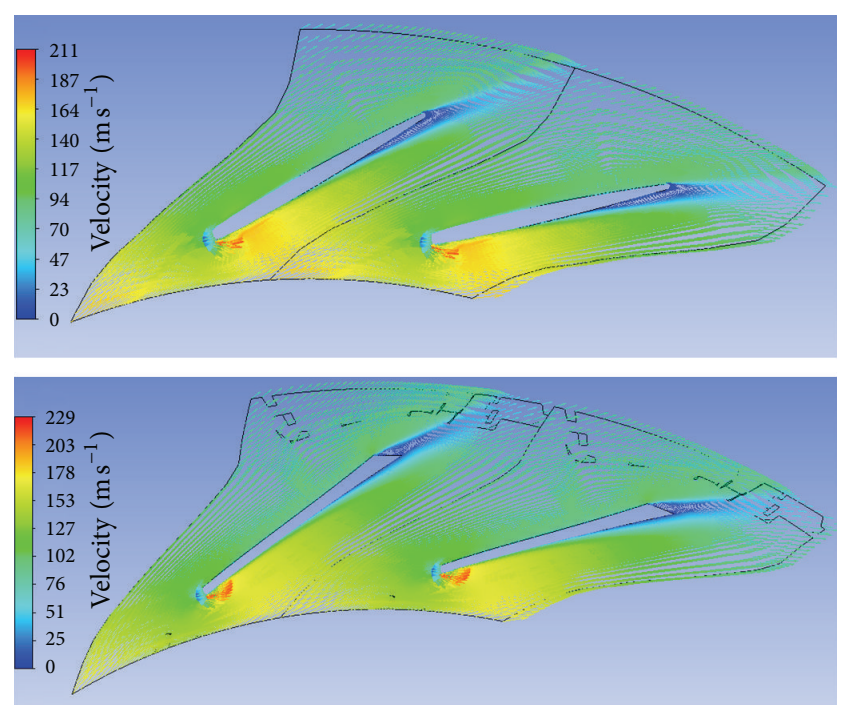

FIGURE 9: Velocity vectors for the midspan plane of VD (top) and $\mathrm{CD}$ (bottom) at $i_{3} \approx 1^{\circ}$.

\section{Conclusions}

The numerical simulation showed the potential availability of the developed channel diffuser design method for the high-performance centrifugal compressor design. Efficiency of the compressor stage may be improved at the range of low mass flow rates because of the flow separation prevention and friction area decrease in the channel diffuser.

The channel diffusers' characteristics are more flat and stable for the positive incidence angles as compared to the vaned diffuser.

At the further stage of the research, the designed channel diffuser will be tested in detail to verify the real advantages of such diffuser and validation of proposed design method.

\section{Nomenclature}

$\begin{array}{ll}r: & \text { Radius } \\ c: & \text { Velocity } \\ \alpha: & \text { Mean flow angle } \\ p: & \text { Pressure } \\ \Delta p=\left(p_{\mathrm{ps}}-p_{\mathrm{ss}}\right): & \text { Pressure difference between surfaces of } \\ & \text { the vane } \\ \rho: & \text { Density } \\ T: & \text { Temperature } \\ \tau: & \text { Blockage factor } \\ \lambda=c / a_{\mathrm{cr}}: & \text { Coefficient of velocity } \\ a_{\mathrm{cr}}: & \text { Critical sonic speed } \\ b: & \text { Width of diffuser } \\ \bar{m}: & \text { Mass flow rate } \\ M: & \text { Angular momentum } \\ z_{v}: & \text { Quantity of vanes } \\ \gamma: & \text { Ratio of specific heats } \\ \pi(\lambda): & \text { Gas dynamic function of pressure } \\ \varepsilon(\lambda): & \text { Gas dynamic function of density } \\ \delta_{v}: & \text { Vane thickness }\end{array}$

$\begin{array}{ll}\delta^{\prime}: & \text { Modified vane thickness } \\ \delta^{*}: & \text { Displacement thickness of boundary layer } \\ \delta^{* *}: & \text { Momentum thickness of boundary layer } \\ \sum \delta^{*}: & \text { Total displacement thickness of boundary } \\ & \text { layers } \\ l: & \text { Vane centerline length coordinate } \\ L: & \text { Vane centerline length } \\ \bar{l}=l / L: & \text { Relative length } \\ \bar{\delta}^{* *}=\delta^{* *} / L: & \text { Relative momentum thickness } \\ H_{s}: & \text { Boundary layer shape parameter } \\ f_{s}: & \text { Boundary layer shape parameter } \\ \nu: & \text { Kinematic viscosity } \\ R: & \text { Gas constant } \\ D: & \text { Diameter } \\ \zeta: & \text { Total pressure loss coefficient } \\ C_{p}: & \text { Pressure recovery coefficient. }\end{array}$

\section{Subscripts}

$\begin{array}{ll}\text { in, 3: } & \text { Diffuser inlet } \\ \text { out, 4: } & \text { Diffuser outlet } \\ \text { ps: } & \text { Pressure surface of the vane } \\ \text { ss: } & \text { Suction surface of the vane } \\ r: & \text { Radial direction } \\ \text { cr: } & \text { Critical } \\ v: & \text { Vane } \\ \text { 1: } & \text { Initial condition of the boundary layer } \\ *: & \text { Stagnation parameter } \\ s: & \text { Preseparation condition. }\end{array}$

\section{References}

[1] L. R. Reneau, J. P. Johnston, and S. J. Kline, "Performance and design of straight, two-dimensional diffusers," Journal of Basic Engineering, vol. 89, no. 1, pp. 141-150, 1967.

[2] P. W. Runstadler Jr. and R. C. Dean Jr., "Straight channel diffuser performance at high inlet mach numbers," Journal of Basic Engineering, vol. 91, no. 3, pp. 397-412, 1969.

[3] F. Kano, N. Tazawa, and Y. Fukao, "Aerodynamic performance of large centrifugal compressors," Journal of Engineering for Power-Transactions of the ASME, vol. 104, no. 4, pp. 796-804, 1982.

[4] W. W. Clements and D. W. Artt, "The influence of diffuser channel length-width ratio on the efficiency of a centrifugal compressor," Journal of Power and Energy, vol. 202, no. 1988, pp. 163-169, 1988.

[5] B. S. Stratford, "An experimental flow with zero skin friction throughout its region of pressure rise," Journal of Fluid Mechanics, vol. 5, no. 1, pp. 17-35, 1959.

[6] R. H. Liebeck, "A class of airfoils designed for high lift in incompressible flow," Journal of Aircraft, vol. 10, no. 10, pp. 610617, 1973.

[7] D. E. Hobbs and H. D. Weingold, "Development of controlled diffusion airfoils for multistage compressor application," Journal of Engineering for Gas Turbines and Power, vol. 106, no. 2, pp. 271-278, 1984.

[8] M. Kalinkevych, O. Obukhov, A. Smirnov, and A. Skoryk, "The design of vaned diffusers of centrifugal compressors based on the given velocity distribution," in Proceedings of the 7th 
International Conference on Compressors and their Systems, pp. 61-69, Woodhead Publishing, 2011.

[9] L. G. Loitcyanskii, MekhanIka ZhIdkostI I Gaza [FluId MechanIcs (In RussIan)], Drofa, Moscow, Russia, 2003.

[10] F. Menter, M. Kunitz, and R. Langtry, "Ten years of industrial experience with the SST turbulence model," Journal of Turbulence, Heat and Mass Transfer, vol. 4, pp. 625-632, 2003. 

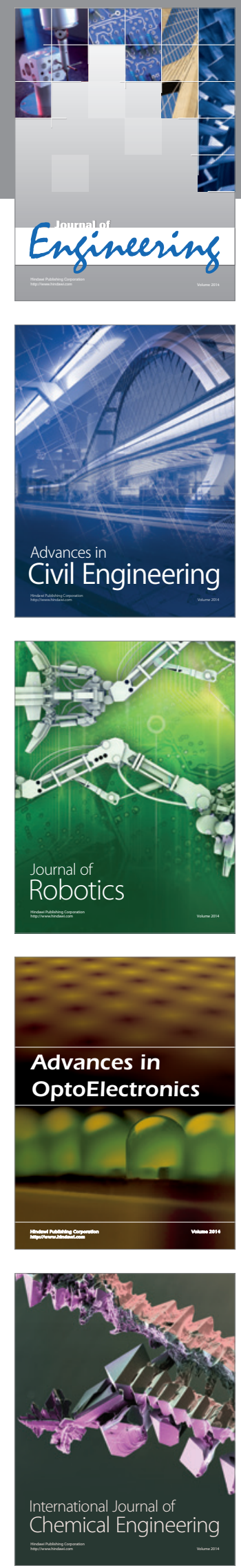

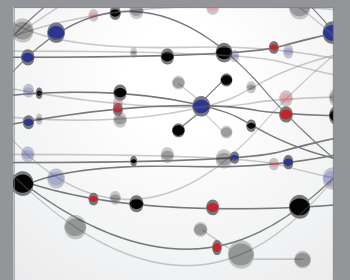

The Scientific World Journal
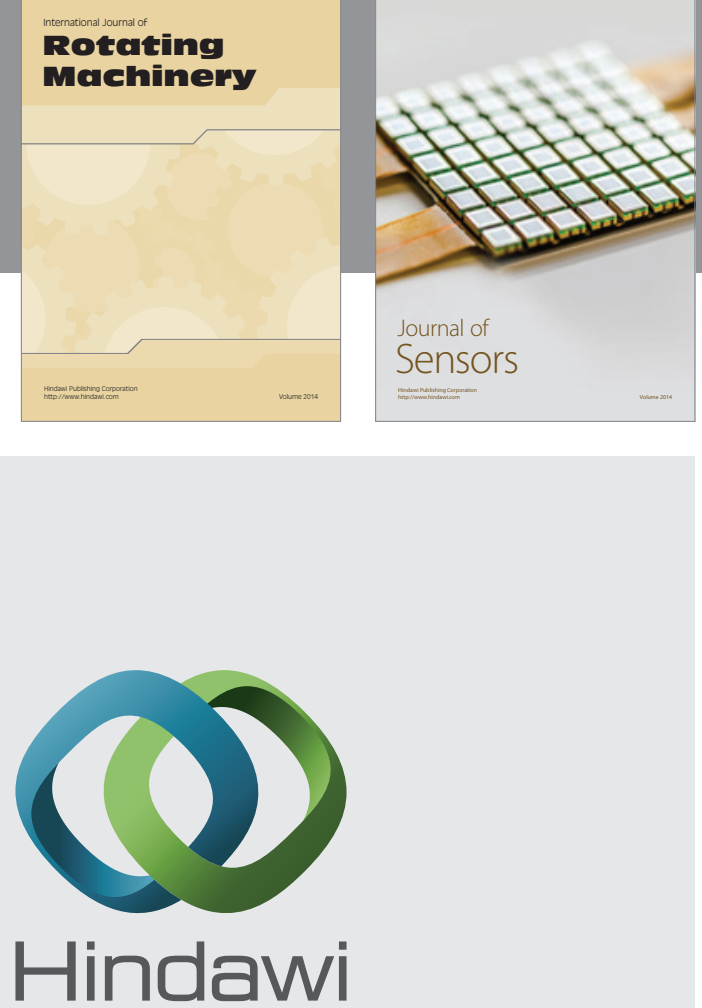

Submit your manuscripts at http://www.hindawi.com
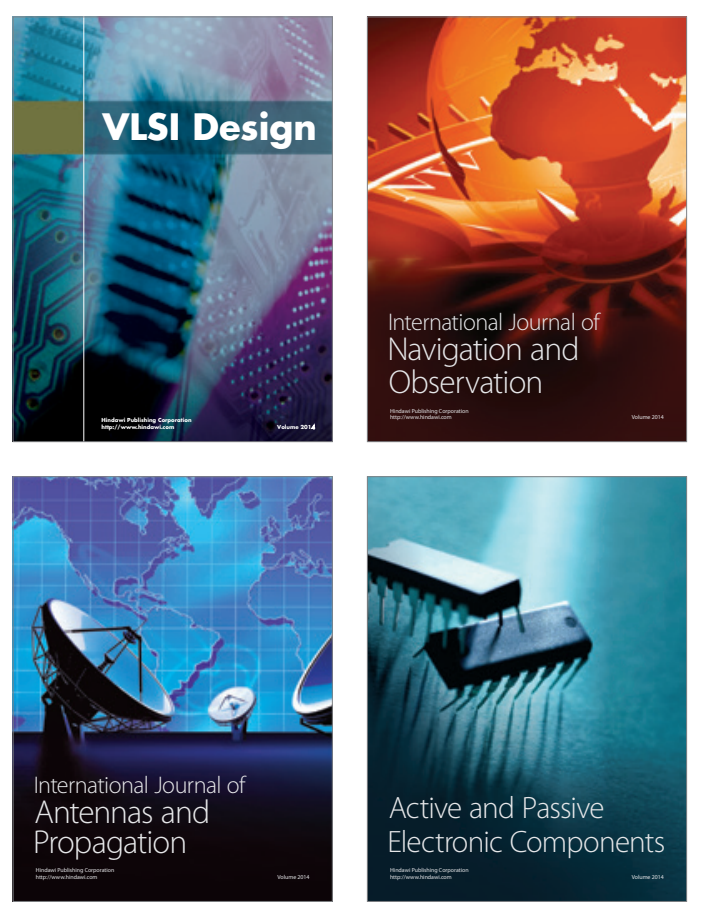
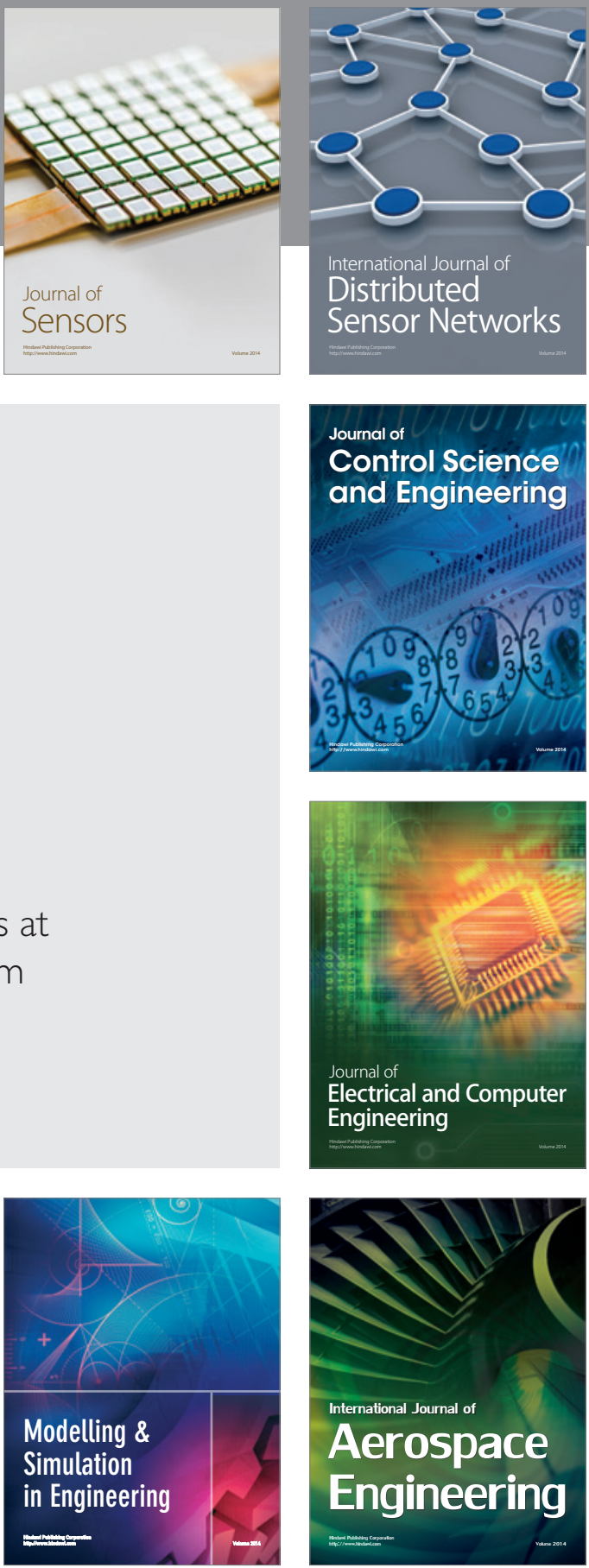

Journal of

Control Science

and Engineering
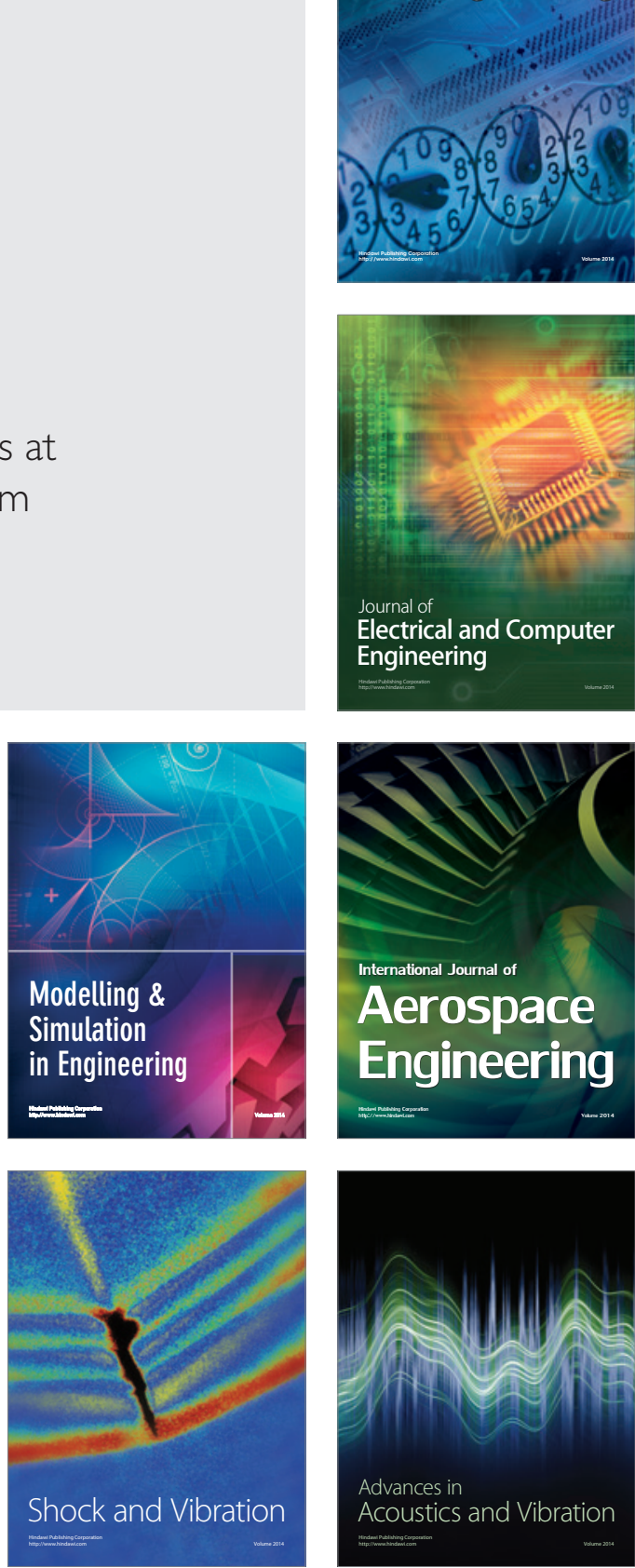